\section{Hodgkin's lymphoma in a man with dilated cardiomyopathy and paraneoplastic ataxia: a therapeutical challenge}

\author{
Elisabetta Abruzzese, ${ }^{1}$ \\ Malgorzata Monika Trawinska, ${ }^{1}$ \\ Achille Gaspardone, ${ }^{2}$ \\ Antonino Morocutti, ${ }^{3}$ \\ Paolo de Fabritiis ${ }^{1}$ \\ ${ }^{1}$ Division of Hematology, ${ }^{2}$ Division of \\ Cardiology, ${ }^{3}$ Division of Neurology, $S$. \\ Eugenio Hospital, Tor Vergata \\ University, Rome, Italy
}

\begin{abstract}
Hodgkin's lymphoma is a cancer of the lymphatic system. We report the case of a man with Hodgkin's lymphoma and cardiomyopathy, for which the dilemma was whether to use the standard protocol - putting the patient at risk of worsening of heart failure, but giving him a good chance of full recovery - or not. The standard protocol was given and the patient made a full recovery without cardiac complications.
\end{abstract}

\section{Introduction}

Hodgkin's lymphoma is a cancer of the lymphatic system that generally has a good prognosis. Indeed, the long-term survival rate of patients in stage I or II is over $85 \%$ while, in more advanced stage, is between $60 \%$ and $75 \%$. Unfavorable prognostic factors are advanced stage, the presence of $B$ symptoms, large masses, involvement of more than 4 lymph node areas, and presence of co-morbidities. ${ }^{1,2}$

In the initial stages, usually 3 to 4 courses of chemotherapy are administered according to the ABVD protocol (Adriamycin, Bleomycin, Vinblastine and Dacarbazine) followed by radiotherapy. Patients with advanced disease (stages IIIB, IV) are treated with 6 courses of chemotherapy. The German Cooperative Group has introduced an intensive polychemotherapy protocol for intermediate to high risk patients (BEACOPP: Bleomycin, Etoposide, Adriamycin, Cyclofosfamide, Vincristine, Procarbazine and Prednisone), which includes 6 to 8 monthly courses administered at standard(s) or increasing dose(s). The selection of the therapeutic protocol is based also on the clinical features of the patient, such as age, pulmonary and cardiac function, and the presence of any concomitant diseases. ${ }^{2,3}$ We report the case of a young adult patient with severe dilated cardiomyopathy, heart failure and neurologic symptoms who developed Hodgkin's lymphoma, in whom the therapeutical challenge was the use of a standard protocol for Hodgkin's lymphoma with an increased risk of worsening heart failure, giving him, however, a good chance of full recovery from the lymphoma.

\section{Case Report}

The patient was a 44-year-old man without any relevant family history. His medical history was unremarkable until September 2007, when he reported pain in his spinal column and right arm, which was treated unsuccessfully with steroids. Radiographs showed the protrusion of multiple discs at several levels and a submandibular lesion $(1 \mathrm{~cm})$ associated with obstruction of a salivary gland. There was no surgical indication and the patient followed a diet and continued symptomatic and steroid treatment. However, pain was not fully under control and after 4 months he developed weakness of the lower limbs (January 2008). One month later, because of worsening asthenia and short of breath associated with jugular congestion and lower limb edema, he was referred to the cardiology outpatient clinic. Transthoracic echocardiography assessment revealed a markedly increased left ventricular volumes and a severely reduced left ventricular ejection fraction estimated at $27 \%$. Radioisotope-left ventriculography (Figure 1) confirmed the reduced left ventricular function and normal myocardial perfusion scan. Coronary angiography showed the absence of angiographically significant coronary artery disease. Treatment with ACE-inhibitor (ramipril $5 \mathrm{mg}$ tid), furosemide (50 mg, tid) and low dose of carvedilol (6.25 mg tid) was initiated with a prompt improvement of cardiovascular symptoms. Two months later the patients was re-hospitalized for follow-up cardiological assessments. Echocardiographic examination revealed a significant improvement of left ventricular function with an ejection fraction estimated at $43 \%$ associated with symptoms improvement. At this time, a lateral cervical lymphadenopathy was reported, but additional diagnostic investigations were not deemed necessary. After few months, the patient complained that the lymphadenopathy had increased in size and a biopsy under sonographic guidance was carried out. The histopathology examination revealed the presence of classi-
Correspondence: Elisabetta Abruzzese, Division of Hematology, S. Eugenio Hospital, Tor Vergata University, P. le dell'Umanesimo 10, 00144, Rome, Italy.

E-mail: elisabetta.abruzzese@uniroma2.it

Key words: Hodgkin's lymphoma; cardiomyopathy; comorbidity; ataxia; heart failure.

Contributions: the authors contributed equally.

Conflict of interest: the authors declare no potential conflict of interest.

Acknowledgements: the authors would thank Bianco Airone Pazienti Onlus for support.

Received for publication: 22 October 2016. Accepted for publication: 21 March 2017.

This work is licensed under a Creative Commons Attribution-NonCommercial 4.0 International License (CC BY-NC 4.0).

(C) Copyright E. Abruzzese et al., 2017

Licensee PAGEPress, Italy

Hematology Reports 2017; 9:6944

doi:10.4081/hr.2017.6944

cal Hodgkin's lymphoma of the nodularsclerosis type and in June 2009 the patient was referred to the hematology outpatient clinic. The CT scan detected confluent pathological lymph nodes in the retropharyngeal, jugular digastric and lateral cervical areas on the right (dimensions: $6 \times 4.5$ $\mathrm{cm})$ and an enlarged spleen $(20 \mathrm{~cm})$ without internal lesions, resulting in IIB stage (due to the recent onset of fever and night sweats). He also had difficulty walking and weakness of the lower limbs together with ataxia. PET scans confirmed increased tracer uptake in the whole right half of the neck, with lymph nodes involvement in the retro pharynx, jugular and latero-cervical but no pathological uptake in the medistinum, spleen or other districts (stage II B).

The therapeutical challenge in this young patient was the simultaneous presence of a severe dilated cardiomyopathy and Hodgkin's symptomatic lymphoma with large masses. Furthermore, he also had clinically important degenerative neuropathy that could represent a paraneoplastic consequence of the lymphoma. In this case, anthracyclines were contraindicated, in view of the definite cardiological risk. However, these drugs represent an important component of all therapeutic regimens designed to induce remission in lymphoma patients. ${ }^{4,5}$ To avoid the risk of under treatment, despite the increased cardiovascular risk, a $2+2$ ABVD courses plus radiotherapy was undertaken monitoring the patient 
closely with repeated echocardiograms. Of note, adriamycin with non-pegylated liposomal doxorubicin was replaced in order to reduce the impact on the cardiac muscle. Although the use of non-pegylated liposomal doxorubicin is an off-label indication in Hodgkin lymphoma, the decision was based both on the published experience in the treatment of non-Hodgkin's lymphomas and on data collected for several years in the treatment of metastatic breast cancer, showing an improved therapeutic index as compared to Adriamycin. ${ }^{6-9}$ The drug is approved in Italy for non-Hodgkin Lymphomas and introduced into daily clinical practice in selected cases. Moreover, the suggested dosages for Hodgkin's lymphoma are lower than those for non Hodgkin's lymphomas $\left(25 \mathrm{mg} / \mathrm{m}^{2}\right.$ instead of $50 \mathrm{mg} / \mathrm{m}^{2}$ ) and the initial stage (II) made it possible to administer only 4 instead of 6 courses.

The patient began treatment in July 2009 after having undergone an examination of the cerebrospinal fluid to detect any cancerous cells in the CNS and/or the presence of antinerve antibodies. Both investigations yielded negative results. During the first infusion, the patient developed a severe allergic reaction with chills and fever, but no hypotension. Bleomycin was identified as the agent that was the most likely cause of the reaction and was stopped; the reaction resolved after the administration of low dose steroids. Subsequent courses were uneventful. After 2 courses, a PET investigation showed absence of uptake. Echocardiography and Doppler parameters remained stable without enzymatic evidence of myocardial damage. Gait also markedly improved with disappearance of ataxia, although some lack of strength persisted. Upon completion of the 4 courses of chemotherapy, a complete remission of the lymphoma was achieved. The patient could walk independently and cardiac status was stable (NYHA Class I) without signs of cardiac failure and with a preserved left ventricular ejection fraction. The patient completed radiotherapy in December 2009 achieving complete remission. At last follow-up (July 2016) the patient feels well, has resumed working and is able to lead an independent life, although he has not fully recovered neurological function. Follow up radioisotope-left ventriculography showed a substantial preservation of left ventricular function at 2 years, stable in the subsequent controls (Figure 1).
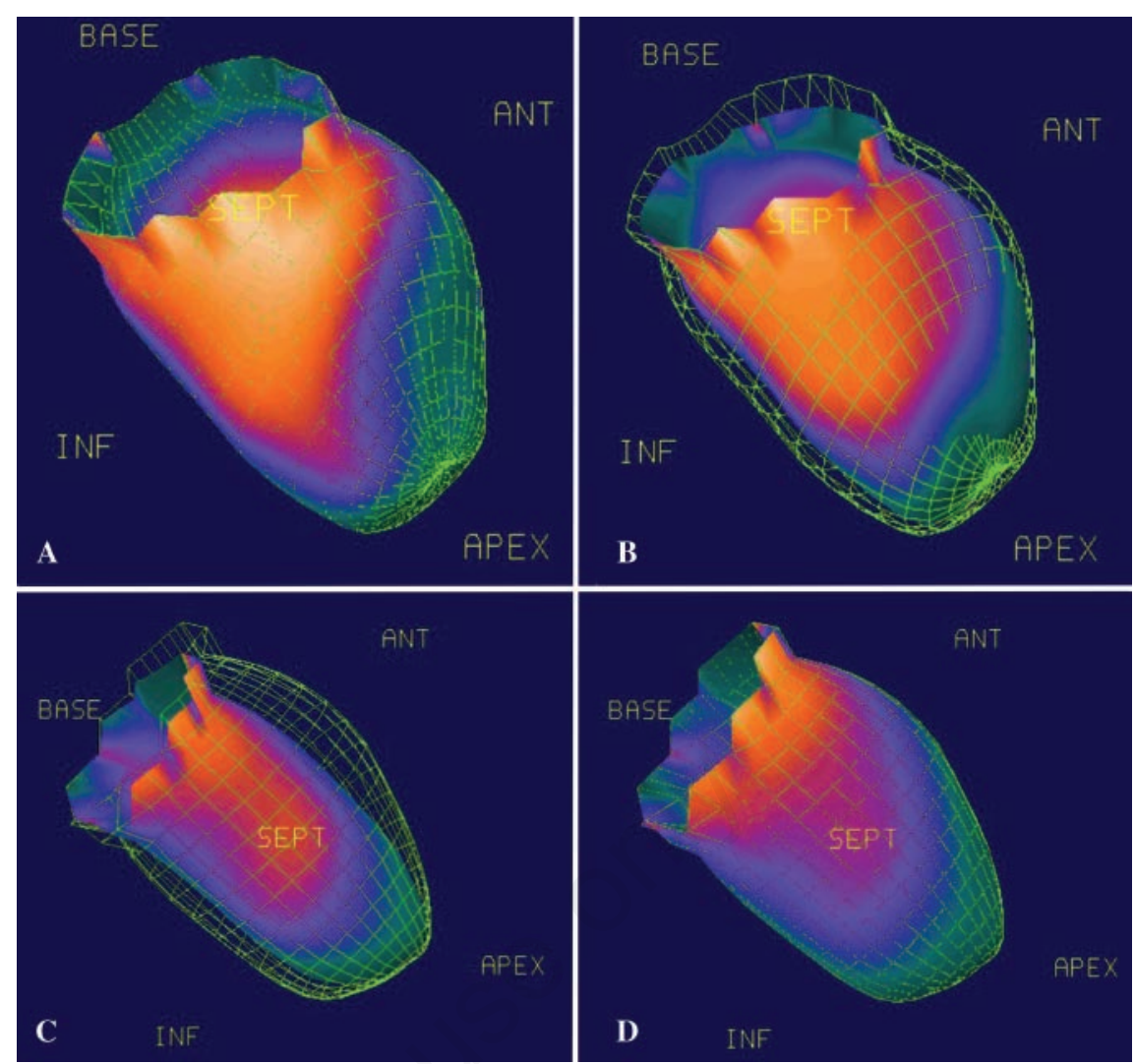

Figure 1. Radioisotope-left ventriculography at symptoms onset (A, diastole and B, systole) and two years later after chemotherapy (C, diastole and $D$, systole).

\section{Discussion and Conclusions}

This is an unusual case of Hodgkin's lymphoma on account of the concomitant cardiomyopathy and paraneoplastic neurologic toxicity. To our knowledge, no such case has been reported in the literature.

Adriamycin and other anthracyclines can produce cardiotoxicity. The risk is directly proportional to the dose received and increases in patients with a history of cardiomyopathy. ${ }^{4,5}$ The presence of a severe form of cardiomyopathy in our patient made the use of anthracyclines, which are essential for the treatment of lymphoma, contraindicated. Non-pegylated liposomal doxorubicin is an anthracycline that is not released in cardiac muscle on account of its liposomal structure and this is thought to reduce its cardiotoxicity. Cardiac events are significantly less common in patients treated with liposomal doxorubicin as compared to patients treated with the same dose of standard Adriamycin, with a low rate of both clinically overt and subclinical heart failure. ${ }^{10}$ Starting from the well established concept in the elderly population suffering from lymphoma that inadequate treatment in a chemosensitive tumor has a worse outcome than the toxicity of the chemotherapy itself, we decided to carry out the treatment of first choice at standard dosage. Nonpegylated liposomal doxorubicin allowed us to provide the full efficacy of anthracyclines, thus administering otherwise contraindicated standard treatment, even in a patient with important risk factors. This treatment schedule was extremely efficacious yielding a prompt and persistent remission of Hodgkin's lymphoma without any consequence on cardiac function, and also the neurological status rapidly improved.

\section{References}

1. Lister TA, Crowther D, Sutcliffe SB, et al. Report of a committee convened to discuss the evaluation and staging of patients with Hodgkin's disease: Cotswolds meeting. J Clin Oncol 1989;7:1630-6.

2. Brusamolino E, Bacigalupo A, Barosi G, et al. Classical Hodgkin's lymphoma in adults: guidelines of the Italian 
Society of Hematology, the Italian Society of Experimental Hematology, and the Italian Group for Bone Marrow Transplantation on initial work-up, management, and follow-up. Haematologica 2009;94:550-65.

3. Engert A, Eichenauer DA, Dreyling M, on behalf of the ESMO Guidelines Working Group. Hodgkin's lymphoma: ESMO clinical practice guidelines for diagnosis, treatment and follow-up. Ann Oncol 2010;21:v168-71.

4. Hershman DL, McBride RB, Eisenberger A, et al. Doxorubicin, cardiac risk factors, and cardiac toxicity in elderly patients with diffuse B-cell nonHodgkin's lymphoma. J Clin Oncol 2008;26:3159-65.

5. Aapro M, Bernard-Marty C, Brain
EGC, et al. Anthracycline cardiotoxicity in the elderly cancer patient: a SIOG expert position paper. Ann Oncol 2011;22:257-67.

6. Leonard RC, Williams S, Tulpule A, et al. Improving the therapeutic index of anthracycline chemotherapy: focus on liposomal doxorubicin (Myocet). Breast 2009;18:218-24.

7. Dell'Olio M, Scalzulli RP, Sanpaolo G, et al. Non-pegylated liposomal doxorubicin (Myocet $\AA$ ) in patients with poorrisk aggressive B-cell non-Hodgkin lymphoma. Leuk Lymphoma 2011;52:1222-9.

8. Rigacci L, Mappa S, Nassi L, et al. Liposome-encapsulated doxorubicin in combination with cyclophosphamide, vincristine, prednisone and rituximab in patients with lymphoma and concurrent cardiac diseases or pre-treated with anthracyclines. Hematol Oncol 2007;25:196-203.

9. Corazzelli G, Frigeri F, Arcamone M, et al. Biweekly rituximab, cyclophosphamide, vincristine, non-pegylated liposome-encapsulated doxorubicin and prednisone (R-COMP-14) in elderly patients with poor-risk diffuse large Bcell lymphoma and moderate to high life threat impact cardiopathy. $\mathrm{Br} \mathrm{J}$ Haematol 2011;154:579-89.

10. Van Dalen EC, Michiels EM, Caron HN, Kremer LC. Different anthracycline derivates for reducing cardiotoxicity in cancer patients. Cochrane Database Syst Rev 2010;12:CD005006. 